\title{
Expression of mRNA for nutrient transporters in the gastrointestinal tract before and after weaning
}

\author{
H. Hayashi' ${ }^{1}$, T. Yonezawa, T. Kanetani, K. Katoh and Y. Obara \\ Department of Animal Physiology, Postgraduate School of Agricultural Science, \\ Tohoku University \\ Tsutsumidori, Aoba-ku, Sendai 981-8555, Japan
}

\begin{abstract}
We investigated mRNA expression of the glucose transporter, SGLT1, and the long-chain fatty acids translocase, CD36, in the gastrointestinal tract of cattle and goats before and after weaning. SGLT1 and CD36 expression in the jejunum was highest in the gastrointestinal tract before weaning, but it decreased in the small intestine after weaning in both species. From these results, we conclude that the jejunum is likely to be the major absorption site for glucose and long-chain fatty acids before weaning, and the expression of both types of transporters decrease in cattle and goats after weaning.
\end{abstract}

KEY WORDS: sglt1, cd36, gastrointestinal tract, mRNA expression

\section{INTRODUCTION}

In the mammalian digestive tract, nutrients are absorbed by various types of transporters. It is thought that glucose and long-chain fatty acids are transported by SGLT1 and CD36, respectively. Before weaning, ruminants digest ingested milk in the same way as do monogastric animals. On the other hand, after weaning, the ruminant has a symbiotic relationship with bacteria that develops along with the morphological structure of the rumen. Carbohydrates in feed are fermented to volatile fatty acids such as acetic, propionic and butyric acids by the activities of ruminal microbes, and these volatile fatty acids are absorbed from the rumen. This means that glucose flowing into the small intestine decreases as the rumen develops. Furthermore, long chain fatty acids hardly reach the small intestine following weaning. Therefore, the expression of nutrient transporters has to change to meet this fore-stomach development. In the ruminant, SGLT1

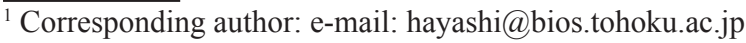


is reported to decrease after weaning (Dyer et al., 1997), but it is unknown whether weaning affects the expression of CD36. In addition, it is not known whether SGLT1 and CD36 expression in the gastrointestinal tract is commonly changed after weaning across species. In this study, therefore, we investigated the expression of nutrient transporters in the gastrointestinal tracts of cattle and goats before and after weaning.

\section{MATERIAL AND METHODS}

Holstein cattle and Saanen goats before and after weaning (4 and 13weeks old) were used. Animals were weaned at 6 weeks of age. Epithelial tissues of the rumen, reticulum, omasum, abomasum, duodenum, jejunum, ileum and colon (cattle only) were collected immediately after slaughter. Total RNA was extracted with TRIzol Reagent (Invitrogen, Carlsbad, CA, USA), and cDNA was synthesized from $5 \mu \mathrm{g}$ of total RNA using SuperScriptII First-Strand Synthesis System (Invitrogen, Carlsbad, CA, USA). SGLT1, CD36 and GAPDH (internal control) were measured by real-time PCR using the LightCycler system (Roche Diagnostics, Mannheim, Germany).

\section{RESULTS}

In all tissues investigated, the expression of SGLT1 and CD36 was confirmed. In both species, jejunum SGLT1 expression was significantly greater than all other tissues before weaning. In goats, the duodenum expression was the second greatest (Table1). Weaning did not change the expression in the fore-stomachs. However, the cattle intestinal SGLT1 expression significantly decreased, and the goat intestinal expression tended to decrease after weaning.

Table 1. SGLT1 and CD36 expression in the goat gastrointestinal tract before and after weaning

\begin{tabular}{|c|c|c|c|c|}
\hline \multirow{2}{*}{ Sample } & \multicolumn{2}{|c|}{ SGLT1 $^{1}$} & \multicolumn{2}{|c|}{$\mathrm{CD}^{2} 6^{2}$} \\
\hline & before & after & before & after \\
\hline Rumen & $0.0115 \pm 0.0066^{\mathrm{c}}$ & $0.0030 \pm 0.0012^{\mathrm{d}}$ & $0.0053 \pm 0.0026^{\mathrm{cd}}$ & $0.0004 \pm 0.0001^{\mathrm{c}^{* *}}$ \\
\hline Reticulum & $0.0055 \pm 0.0034^{c}$ & $0.0034 \pm 0.0011^{\mathrm{d}}$ & $0.0075 \pm 0.0073^{\text {cd }}$ & $0.0028 \pm 0.0007^{c}$ \\
\hline Omasum & $0.0062 \pm 0.0013^{\mathrm{c}}$ & $0.0023 \pm 0.0008^{\mathrm{d}^{*}}$ & $0.0010 \pm 0.0002^{\mathrm{d}}$ & $0.0011 \pm 0.0005^{\mathrm{c}}$ \\
\hline Abomasum & $0.0087 \pm 0.0067^{\mathrm{c}}$ & $0.0013 \pm 0.0006^{\mathrm{d}}$ & $0.0439 \pm 0.0160^{c}$ & $0.0223 \pm 0.0082^{b}$ \\
\hline Duodenum & $0.1528 \pm 0.0519^{b}$ & $0.0319 \pm 0.0148^{b \dagger}$ & $0.2068 \pm 0.0652^{b}$ & $0.0504 \pm 0.0295^{\mathrm{a}^{* *}}$ \\
\hline Jejunum & $0.2180 \pm 0.0740^{\mathrm{a}}$ & $0.0598 \pm 0.0173^{\mathrm{a \dagger}}$ & $0.3241 \pm 0.0860^{\mathrm{a}}$ & $0.0179 \pm 0.0072^{\mathrm{b}^{* *}}$ \\
\hline Ileum & $0.0316 \pm 0.0222^{\mathrm{c}}$ & $0.0164 \pm 0.0091^{\mathrm{c}}$ & $0.0035 \pm 0.0020^{\mathrm{cd}}$ & $0.0011 \pm 0.0006^{\mathrm{c}}$ \\
\hline
\end{tabular}

${ }^{1}$ mean \pm S.E. $\left(\right.$ SGLT1/GAPDH), ${ }^{2}$ mean \pm S.E. $($ CD $36 /$ GAPDH $)$

a,b,c,d values without a common superscript letter were significantly different in the same column: $\mathrm{P}<0.05$

${ }^{* * *, \dagger}$ significance of difference between before and after weaning *- $\mathrm{P}<0.05 ; * *-\mathrm{P}<0.01 ; \dagger-\mathrm{P}<0.1$ 
In cattle and goat jejunum, CD36 expression was significantly greater than all other tissues before weaning. In the goat duodenum, the expression was the second greatest. In cattle, expression significantly decreased after weaning. The expression in the rumen, duodenum and jejunum of goats significantly decreased after weaning, the expression in the duodenum and jejunum being markedly reduced.

\section{DISCUSSION}

In the lactating bovine, Zhao et al. (1998) reported SGLT1 expression in various gastrointestinal tracts. However, the distribution of SGLT1 expression before weaning was not identified in the ruminant. In this study, we have demonstrated that SGLT1 is expressed in various gastrointestinal tracts before weaning. In the jejunum, the expression was greatest before weaning. Accordingly it is suggested that the jejunum is the main glucose absorption site in both species before weaning, and that glucose is transported in the duodenum of goats before weaning because the expression in duodenum was greater before weaning than after.

It is thought that long-chain fatty acids (LCFA) are transported by CD36. CD36 is expressed in rat intestinal brush border membranes and is increased on a high-fat diet (Poirier et al., 1996). In addition, Chen et al. (2001) reported that the jejunum CD36 expression was greatest in the gastrointestinal tract and increased with growth in rats. In this study, the jejunum expression was greatest in both species before weaning. Accordingly it is suggested that the jejunum is the main LCFA absorption site before weaning. This also suggests that the duodenum of goats transports LCFA before weaning because the duodenum expression was greater before weaning than after. It is suggested that the reason why the intestinal expression sharply decreases after weaning is that fats are not generally included in diets after weaning. Therefore, it is suggested that CD36 expression decreased in a mechanism similar to that for SGLT1, because of a decreased nutrient flow into the small intestine.

\section{CONCLUSIONS}

In cattle and goats, before weaning, greater SGLT1 expression in the jejunum suggests that the jejunum is the main absorption site for glucose, but a difference in the SGLT1 expression exists in both species. Before weaning, the highest jejunum CD36 expression suggests that the jejunum is the main LCFA absorption site. The decrease in intestinal CD36 expression after weaning suggests that the LCFA transporter, as for the glucose transporter, may depend on nutrient inflow into the small intestine. 


\section{REFERENCES}

Dyer J., Barker P.J., Shirazi-Beechey S.P., 1997. Nutrient regulation of the intestinal $\mathrm{Na}^{+} /$glucose co-transporter (SGLT1) gene expression. Biochem. Biophys. Res. Commun. 230, 624-629

Zhao F., Okine E.K., Cheeseman C.I., Shirazi-Beechey S.P., Kennelly J.J., 1998. Glucose transporter gene expression in lactating bovine gastrointestinal tract. J. Anim. Sci. 76, 2921-2929

Poirier H., Degrace P., Niot I., Bernard A., Besnard P., 1996. Localization and regulation of the putative membrane fatty-acid transporter (FAT) in the small intestine comparison with fatty acidbinding proteins (FABP). Eur. J. Biochem. 238, 368-373

Chen M., Yang Y., Braunstein E., Georgeson K.E., Harmon C.M., 2001. Gut expression and regulation of FAT/CD36: possible role in fatty acid transport in rat enterocytes. Amer. J. Physiol. - Endocrinol. Met. 281, E916-E923 